\title{
CME-Beitrag
}

Zeitschrift für Der Notarzt

\section{Gynäkologische Notfälle}

\author{
A. Strauss ${ }^{1}$, L. Sanders ${ }^{1}$, J.-T. Gräsner ${ }^{2}$, H. Ohnesorge ${ }^{2}$ \\ ${ }^{1}$ Klinik für Gynäkologie und Geburtshilfe und Michaelis Hebammenschule, \\ Universitätsklinikum Schleswig-Holstein, Campus Kiel, Christian-Albrechts-Universität \\ 2 Klinik für Anästhesiologie und Operative Intensivmedizin, Universitätsklinikum \\ Schleswig-Holstein, Campus Kiel, Christian-Albrechts-Universität
}

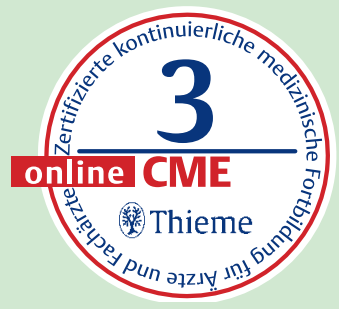




\section{Gynäkologische Notfälle}

\section{Gynaecological Emergencies}

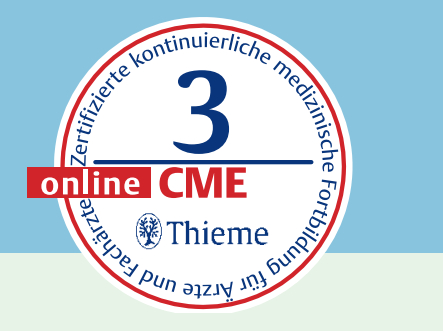

\section{Schlüsselwörter \\ gynäkologischer Notfall \\ vaginale Blutung \\ - Unterbauchschmerzen \\ - akutes Abdomen \\ - ektope Schwangerschaft}

\section{Keywords}

- gynecologic emergencies

- vaginal bleeding

- abdominal pain

- acute abdomen

- ectopic pregnancy
VNR

2760512013141213745

\section{Bibliografie}

Dol http://dx.doi.org/

10.1055/s-0033-1349533

Notarzt 2013; 29:

219-230

(c) Georg Thieme Verlag KC

Stuttgart · New York

ISSN 0177-2309

\section{Korrespondenzadresse}

Univ.-Prof. Dr. med.

\section{Alexander Strauss}

Klinik für Gynäkologie und Geburtshilfe und Michaelis Hebammenschule, Universitätsklinikum Schleswig-Holstein, Campus Kiel, Christian-AlbrechtsUniversität

Arnold-Heller-Straße 3, Gebäude 24 24105 Kiel

Alexander.Strauss@uksh.de
A. Strauss ${ }^{1}$, L. Sanders ${ }^{1}$, J.-T. Gräsner ${ }^{2}$, H. Ohnesorge ${ }^{2}$

\section{Zusammenfassung}

$\nabla$

Die Frauenheilkunde ist - wie auch andere medizinische Disziplinen - durch unvorhersehbar auftretende und akut verlaufende Krankheitsbilder geprägt. Notfallmäßige Krankenhauseinweisungen gynäkologischer Patientinnen erfolgen am häufigsten wegen vaginalen Blutungen (z.B. gestörte Frühschwangerschaft, zyklische Blutungsstörungen, gynäkologische Malignome, Verletzungen), akuter Unterbauchschmerzen bis hin zum akuten Abdomen (u. a. durch Ovarialzysten mit bzw. ohne Komplikationen: Einblutung, Ruptur, Torsion, Infektion, Abszess) oder ektoper Schwangerschaften. Bei stark eingeschränkten präklinischen Diagnose- und Therapiemöglichkeiten ist neben allgemeinen Maßnahmen zur kardiopulmonalen Stabilisierung (ABCDE-Algorithmus ggf. ein situationsadaptiertes Schmerzmanagement während des Transports zu etablieren. Hierbei stellt sich bei der Patientin im reproduktionsfähigen Alter ( $<45$ Jahre) stets die Frage nach einer Schwangerschaft als einer mittelbaren oder unmittelbaren Einflussgröße auf die Notfallsituation.

\section{Abstract \\ $\nabla$}

Corresponding to other clinical disciplines emergencies in gynecology are characterized by an unpredictable onset and acute course. The main reasons for emergency inpatient treatments in gynecological patients are vaginal bleeding (e.g. miscarriage in early pregnancy, bleeding disorders, gynecologic cancer, trauma) abdominal pain with or without signs of an acute abdomen (e.g. ovarian cysts \pm complications: bleeding, rupture, torsion, infection) or ectopic pregnancies. Due to restricted preclinical diagnostic and therapeutic options the emergency treatment is limited to stabilization of the cardiopulmonary system (ABCDE-algorithm) and effective pain therapy. Thus, especially in an emergency setting, a focused medical history is essential and the possibility of a pregnancy in gynecological patients aged $<45$ years must be kept in mind.

\footnotetext{
${ }^{1}$ Klinik für Gynäkologie und Geburtshilfe und Michaelis Hebammenschule, Universitätsklinikum Schleswig-Holstein, Campus Kiel, Christian-Albrechts-Universität

${ }^{2}$ Klinik für Anästhesiologie und Operative Intensivmedizin, Universitätsklinikum Schleswig-Holstein, Campus Kiel, Christian-Albrechts-Universität
} 
Tab. 1 Kernaussagen: präklinische gynäkologische Notfälle [16].

\begin{tabular}{|c|c|c|c|c|}
\hline Diagnose & Symptome & Befunde & Maßnahmen & $\begin{array}{l}\text { Was ist zu beachten? } \\
\text { weitere Differenzial- } \\
\text { diagnosen }\end{array}$ \\
\hline Ovarialtorsion & $\begin{array}{l}\text { akuter, stechender, einseiti- } \\
\text { ger Unterbauchschmerz, } \\
\text { bei intermittierender Torsion } \\
\text { u. U. kolikartiger Schmerz, } \\
\text { Übelkeit, Erbrechen }\end{array}$ & $\begin{array}{l}\text { negativer Schwangerschaftstest, } \\
\text { palpable (teigige Schwellung) im Ad- } \\
\text { nexbereich, Fieber und Leukozytose } \\
\text { bei Nekrose des Ovars, Dopplersono- } \\
\text { grafie: zunächst venöse später arte- } \\
\text { rielle Flussveränderungen (patho- } \\
\text { gnomonisch), ein regelrechtes dopp- } \\
\text { lersonografisches Flussmuster } \\
\text { schließt eine Torsion jedoch nicht aus }\end{array}$ & $\begin{array}{l}\text { frühzeitige Diagnosesicherung und } \\
\text { Therapie durch Laparoskopie, Ver- } \\
\text { such der Retorquierung auch bei ma- } \\
\text { kroskopisch wenig hoffnungsvollen } \\
\text { Befunden, mindestens } 24 \mathrm{~h} \text { stationä- } \\
\text { re Überwachung post Op, Relaparo- } \\
\text { skopie bei Therapieversagen (Anstieg } \\
\text { der Entzündungsparameter, Fieber, } \\
\text { keine Beschwerdebesserung) }\end{array}$ & $\begin{array}{l}\text { Ovarialzystenruptur, } \\
\text { Tuboovarialabszess, } \\
\text { Extrauteringravidität }\end{array}$ \\
\hline $\begin{array}{l}\text { Ovarialzysten- } \\
\text { ruptur }\end{array}$ & $\begin{array}{l}\text { akuter Schmerzbeginn, } \\
\text { Abwehrspannung, } \\
\text { Volumenmangel(-schock) }\end{array}$ & $\begin{array}{l}\text { negativer Schwangerschaftstest, } \\
\text { Raumforderung in einem Adnexbe- } \\
\text { reich, (mäßig viel) freie Flüssigkeit im } \\
\text { Douglas'schen Raum }\end{array}$ & $\begin{array}{l}\text { Schmerztherapie, Kontrolle der hä- } \\
\text { modynamischen Parameter und des } \\
\text { Hb-Werts, ggf. Behandlung eines } \\
\text { Volumenmangelschocks, Diagnose- } \\
\text { sicherung und Therapie durch Lapa- } \\
\text { roskopie bei schwerem Krankheits- } \\
\text { verlauf oder diagnostischer Unsi- } \\
\text { cherheit }\end{array}$ & $\begin{array}{l}\text { Tuboovarialabszess, } \\
\text { Ovarialtorsion, } \\
\text { Extrauteringravidität }\end{array}$ \\
\hline $\begin{array}{l}\text { Tuboovarial- } \\
\text { abszess }\end{array}$ & $\begin{array}{l}\text { Unterbauchschmerz } \\
\text { (u.U. schleichender Beginn), } \\
\text { Portioschiebeschmerz }\end{array}$ & $\begin{array}{l}\text { Fieber, Leukozytose, erhöhte Entzün- } \\
\text { dungswerte, pathologische vaginale } \\
\text { Flora, nachgewiesene Infektion mit } \\
\text { Neisseria gonorrhoeae oder Chlamy- } \\
\text { dia trachomatis }\end{array}$ & $\begin{array}{l}\text { intravenöser Zugang und Flüssig- } \\
\text { keitssubstitution, operative Abszess- } \\
\text { spaltung und Drainage (LSK), anti- } \\
\text { biotische Therapie (z. B. Ampicillin } \\
2 \mathrm{~g} 4 \text {-mal tgl. + Clindamycin 1,2g } \\
\text { 2-mal tgl. i.v. oder Cefuroxim + Doxy- } \\
\text { cyclin + Metronidazol), wenn keine } \\
\text { primär operative Sanierung: klare } \\
\text { Definition von konservativen Thera- } \\
\text { pieversagern: kein Abfiebern inner- } \\
\text { halb von } 72 \text { Stunden oder Vergröß- } \\
\text { erung des Abszesses unter Therapie }\end{array}$ & $\begin{array}{l}\text { Ovarialtorsion, } \\
\text { Extrauteringravidität, } \\
\text { Ovarialzystenruptur }\end{array}$ \\
\hline $\begin{array}{l}\text { Extrauterin- } \\
\text { gravidität }\end{array}$ & $\begin{array}{l}\text { sekundäre Amenorrhö 6-8 } \\
\text { Wochen, schwache vaginale } \\
\text { Blutung, ggf. akutes Abdo- } \\
\text { men } \\
\text { Tubarabort: wellenförmiger } \\
\text { einseitiger Unterbauch- } \\
\text { schmerz, } \\
\text { Tubarruptur: stärkster, plötz- } \\
\text { licher Unterbauchschmerz }\end{array}$ & $\begin{array}{l}\text { Schwangerschaftstest positiv, aufge- } \\
\text { lockerter, für das Schwangerschafts- } \\
\text { alter zu kleiner Uterus, } \\
\text { Sonografie: leeres Cavum uteri, } \\
\text { (ringförmige) Raumforderung im } \\
\text { Adnexbereich, freie Flüssigkeit Dou- } \\
\text { glas'scher Raum oder betont einsei- } \\
\text { tig im Adnexbereich }\end{array}$ & $\begin{array}{l}\text { intravenöser Zugang und Flüssig- } \\
\text { keitssubstitution, Stabilisierung der } \\
\text { Vitalparameter, Bestimmung von } \beta \text { - } \\
\text { hCG, Blutbild, Gerinnung (Kreuz- } \\
\text { blut), Laparoskopie (wenn möglich } \\
\text { organerhaltendes Vorgehen, bei Re- } \\
\text { zidiv oder zerstörter Tubenarchitek- } \\
\text { tur und makroskopisch einwandfreier } \\
\text { Gegenseite bzw. bei operativer Not- } \\
\text { wendigkeit } \rightarrow \text { Salpingektomie, alter- } \\
\text { nativ medikamentöse Therapie } \\
\text { [MTX]) oder Observanz, Rhesuspro- } \\
\text { phylaxe [falls erforderlich]) }\end{array}$ & $\begin{array}{l}\text { Ovarialtorsion, } \\
\text { Tuboovarialabszess, } \\
\text { Ovarialzystenruptur }\end{array}$ \\
\hline
\end{tabular}

Hb: Hämoglobin, LSK: Laparoskopie, MTX: Methotrexat, hCG: humanes Choriongonadotropin

Wie alle medizinischen Disziplinen ist auch die Frauenheilkunde durch unvorhersehbar auftretende und akut verlaufende Krankheitsbilder geprägt. Diese akuten Gesundheitsstörungen können Frauen zu allen Phasen ihres Lebens zur Notfallpatientin machen. Die Kernaussagen zu gynäkologischen Notfällen sind in $\odot$ Tab. 1 zusammengefasst [1].

\section{Vaginale gynäkologische Blutungen}

Schwerwiegende gynäkologische Blutungen kommen in jedem Lebensalter vor. $12 \%$ aller Krankenhauseinweisungen in eine gynäkologische Abteilung erfolgen wegen verstärkter vaginaler Blutungen. Hiervon erfordert nur ein kleiner Prozentsatz eine akute frauenärztliche Intervention. Das Risiko eines jeden starken Blutverlusts ist Anämie und in der Folge ein hämorrhagischer Schock. Das Geschehen entwickelt sich sequenziell und ist durch zeitnahe adäquate Intervention reversibel. Zum Notfall führende vaginale Blutungen können in unterschiedlichen Lebensaltern der Frau akut oder chronisch, 1-malig oder rezidivierend auftreten. Behandlungserfordernis ergibt sich aus der vorliegenden Symptomatik, der Ursache der Blutung und deren Komplikationsmöglichkeiten (z.B. Kreislaufwirksamkeit des Blutverlusts). Sofortmaßnahmen

- $12 \%$ aller Krankenhauseinweisungen in eine gynäkologische Abteilung erfolgen wegen verstärkter vaginaler Blutungen. 
- Sofortmaßnahmen sind ausreichende Oxygenierung, Volumenwiederherstellung und Substitution von Blutbestandteilen.

- Häufige Ursache einer Blutung im Rahmen einer gestörten Frühschwangerschaft ist ein drohender oder stattfindender Abort.

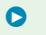
Bei überregelstarker uteriner Blutung ist die fraktionierte Kürettage als gleichzeitig diagnostische und therapeutische Maßnahme das Mittel der 1. Wahl.

- Für die präklinische Versorgung ergibt sich Interventionsbedarf in Abhängigkeit des Blutdrucks der Patientin.

- Verletzungen im Bereich des äußeren bzw. inneren Genitales sind selten Ursachen akuter vaginaler Blutungen.
- Das Spektrum der Symptomatik ist bei Ovarialzysten sehr variabel. sind eine ausreichende Oxygenierung der Patientin, Wiederherstellung eines ausreichenden zirkulierenden Volumens (kristalloide und kolloidale oder hyperonkotisch-hyperosmolare Infusionslösungen) sowie in der Klinik die Substitution von Blutbestandteilen [2].

\section{Häufige Ursachen}

Häufige Ursache einer Blutung im Rahmen einer gestörten Frühschwangerschaft ist der drohende oder stattfindende Abort (Abortus imminens, Abortus incipiens, Abortus incompletus). Daher ist bei einer blutenden Patientin im reproduktionsfähigen Alter stets ein Schwangerschaftstest oder ab einem Gestationsalter > 6-7 SSW (Schwangerschaftswochen) im Kliniksetting ein sonografischer Schwangerschaftsausschluss zu führen [3, 4]. Bei nachgewiesen gestörter, intrauterin angelegter Schwangerschaft ist zur Minimierung des Blutverlusts eine Abortkürettage durchzuführen. Die Ursachen zyklischer gynäkologischer Blutungsstörungen (Hypermenorrhö, Polymenorrhö, Menorrhagie, Metrorrhagie) können anatomischer (z.B. Uterus myomatosus) oder endokriner (Zyklusstörung, Follikelpersistenz) Natur sein. Die Behandlung kann dabei kausal oder symptomatisch erfolgen. Bei überregelstarker uteriner Blutung ist die fraktionierte Kürettage als gleichzeitig diagnostische und therapeutische Maßnahme der 1. Wahl anzuwenden. Zur Vermeidung weiterer Blutungen bzw. Rezidive sind in Abhängigkeit von individuellen Patientinnencharakteristika medikamentöse Therapieansätze zu wählen. Bei wiederholten Blutungsereignissen, frustraner konservativer Therapie und abgeschlossener Familienplanung kann zur operativen Sanierung der Blutungsursache ein organerhaltendes Vorgehen (hysteroskopische Endometriumablation) oder die Hysterektomie erwogen werden. Alternativ oder ergänzend kommen medikamentöse Therapieansätze infrage. Endokrin wird die uterine Blutung unter anderem mit Gestagenpräparaten behandelt. Diese konservative Therapievariante ist dabei allerdings bevorzugt der Nichtnotfallsituation vorbehalten. Vaginale Blutungen sind bei gynäkologischen Malignomen, z.B. Zervix-, Endometrium-, Vulva- oder Vaginalkarzinom (beide letztgenannten sehr selten), in der Akutphase durch Tamponade, Kauterisierung, Kryotherapie als Überbrückungsmaßnahme bis zur Operation oder Strahlentherapie zum Stillstand zu bringen. Einen palliativen Behandlungsansatz stellt die Möglichkeit der Gefäßembolisation (interventionelle Radiologie) dar [5].

Für die präklinische Versorgung ergibt sich Interventionsbedarf in Abhängigkeit des Blutdrucks der Patientin. Analog den Empfehlungen für Traumapatienten mit Volumenmangel sollte ein Zielblutdruck von $90 \mathrm{~mm} \mathrm{Hg}$ aufrechterhalten oder erreicht werden. Hierfür sollten initial kristalloide Infusionslösungen (1000 ml Ringer-Lösung) zur Anwendung kommen (Empfehlungsstärke Grad B).

\section{Seltene Ursachen}

Seltenere Ursachen akuter vaginaler Blutungen sind Verletzungen im Bereich des äußeren und bzw. oder inneren Genitales. Um die Ausdehnung der Verletzung einzuschätzen und eine geeignete Behandlungsstrategie auszuwählen, spielt die anamnestische Klärung des Unfallhergangs eine besondere Rolle. Dies ist oftmals bei Kohabitationsverletzungen und bei Verletzungen von Kindern (Missbrauchsvermutung) ausgesprochen schwierig. Bei stumpfen Bauchtraumen können zusätzlich zur äußeren auch intraabdominale Blutungen auftreten. Führendes Symptom ist ein akutes Abdomen kombiniert mit einem sich rasch entwickelnden hämorrhagischen Schock. Sowohl das klinische Bild wie auch die laborchemische und die apparative Akutdiagnostik (Blutbild, Labor, Sonografie, Röntgen, CT [Computertomografie]) erlauben in der Zusammenschau abzuschätzen, wie dringlich eine Operationsindikation ist. Größere Traumata (wie z.B. Pfählungsverletzungen) erfordern dabei ein interdisziplinäres Vorgehen [5, 6]. Notfallmedizinisch erfolgt die Therapie wie bei nicht frauenärztlichen Patienten mit Bauchtraumata. Der Zielblutdruck bei unkontrollierbaren Blutungen sollte bei $90 \mathrm{~mm} \mathrm{Hg}$ liegen (Empfehlungsstärke Grad B).

\section{Akuter Unterbauchschmerz - Akutes Abdomen \\ $\nabla$}

Die Entstehung von Ovarialzysten und deren Einblutung, Torsion oder Ruptur sind häufige gynäkologische Ursachen von akut - als Notfall - auftretenden Unterbauchschmerzen [7, 8].

\section{Ovarialzysten: Einblutung und Ruptur}

Die Einblutung oder in der Folge Ruptur einer ovariellen Zyste kann zum akuten Schmerzereignis durch die erhöhte Wandspannung oder die peritoneale Reizung durch den Zysteninhalt bzw. die nachfolgend einsetzende Blutung aus dem Zystengrund führen. Das Spektrum der Symptomatik kann dabei sehr variabel sein (asymptomatischer Verlauf, Schmerz bei körperlicher Akti- 


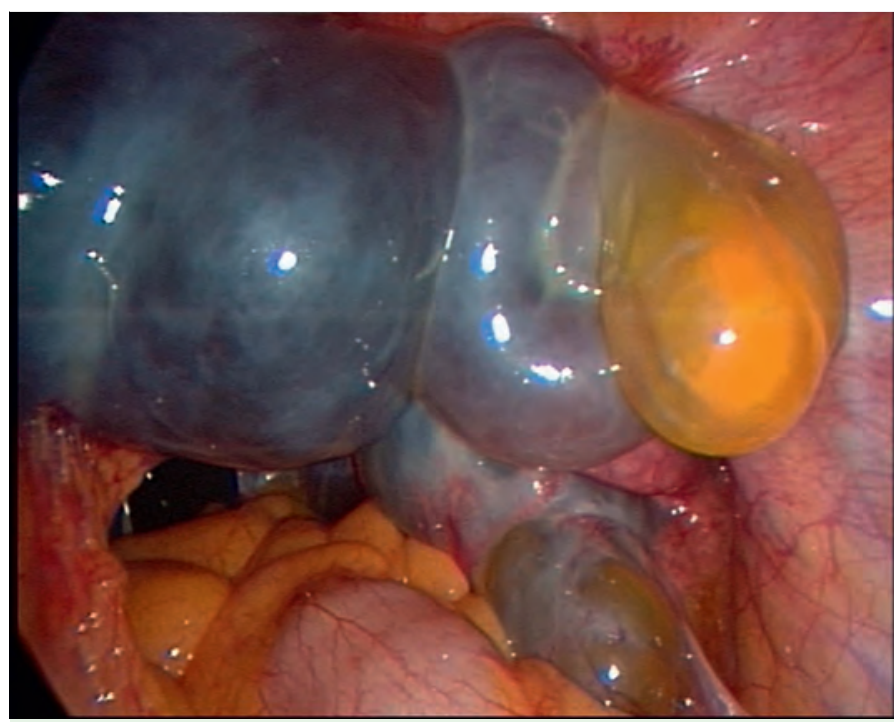

Abb. 1 Laparoskopischer Aspekt einer eingebluteten Ovarialzyste rechts. Symptomatik: Akutes Abdomen bei Spannungsschmerz und peritonealer Reizung (durch Hämatozele im Douglas'schen Raum).

vität z.B. Sport bzw. Geschlechtsverkehr, akut einsetzender, stärkster, einseitiger Unterbauchschmerz). Diagnostisch maßgeblich sind neben dem klinischen Bild im klinischen Verlauf die gynäkologische Palpation und die sonografische Abklärung [4]. Aufgrund fehlender diagnostischer Möglichkeiten im Notarzteinsatz sollten Patientinnen einer fachärztlichen Diagnostik in einer geeigneten Klinik (gynäkologische Fachabteilung vorhanden) zugeführt werden. Anamnestischer Hinweis auf eine Zysteneinblutung und bzw. oder -ruptur kann die Zyklusmitte (14 Tage nach Beginn der letzten Menstruation) sein. Die präklinische Behandlung umfasst die Analgesie und Kreislaufstabilisierung. Die stationäre Behandlung umfasst sowohl eine fortgesetzte Schmerztherapie als auch die Überwachung der klinischen und laborchemischen Parameter (Hämoglobinwert). Eine laparoskopische Exploration ist stets bei instabiler oder sich klinisch verschlechternder Patientin indiziert $(\bullet$ Abb.1). Als weitere Komplikationsmöglichkeit ist bei konservativer Betreuung an eine (zukünftige) Torsion des Ovars zu denken und die Patientin unter dem Prophylaxeaspekt entsprechend zu beraten [8].

\section{Ovarial(zysten)torsion}

Als Ovarialtorsion bezeichnet man die Drehung des Ovars um seinen Aufhängeapparat mit nachfolgender hämorrhagischer Infarzierung. Die Torsion des Ovars ist für 3\% der gynäkologischen Notfalleingriffe, hiervon 15-25\% in der Schwangerschaft, verantwortlich. Pathogenetisch spielt das Volumen der häufig einer Verdrehung des Eierstocks zugrunde liegenden Ovarialzysten $(>5 \mathrm{~cm}$ ) eine wichtige Rolle (sehr große Tumoren verkeilen sich im Becken und neigen nicht mehr zur Torsion). Symptome sind Übelkeit bzw. Erbrechen (70\%) und akute stechende Unterbauchschmerzen $(60 \%)$ mit peritonealer Reizung $(3 \%)$. Fieber und Leukozytose sind seltene Spätzeichen $(<2 \%)$ der irreversiblen Organschädigung (ovarieller Nekrose). Die sichere Diagnose einer Ovarialtorsion erfolgt erst bei der operativen Exploration und entzieht sich somit der notärztlichen Beurteilung. Der Verdacht auf Torsion ergibt sich aus der Anamnese (ruckartige Bewegung, plötzlicher Schmerzbeginn), der Klinik, des Palpationsbefunds (schmerzhafte, teigige Raumforderung im Adnexbereich) und kann in der Klinik durch die sonografische Darstellung des aufgetriebenen Ovars ergänzt werden. Dies gelingt in ca. $40 \%$ der Fälle. Zusätzlich können sich farbdopplersonografische Hinweise für die Abwesenheit des venösen Rückstroms ergeben. Differenzialdiagnostisch ist eine ektope Schwangerschaft, eine Ovarialzystenruptur, ein Tuboovarialabszess, (nekrotisierendes) Myom, eine Endometriose, jedoch ebenso eine Appendizitis oder akute Gastroenteritis abzuwägen [4]. Die Behandlung der Wahl stellt die frühzeitige laparoskopische Intervention dar. Nur die rasche Retorquierung eröffnet die Chance auf Organerhalt (24 $\mathrm{h}$ beobachten) [7]. Als präklinisch entscheidende Stellgröße kann sich somit der zielgerichtete Transport an eine Klinik mit gynäkologischer Versorgungsmöglichkeit erweisen.

\section{Tuboovarialabszess}

Der Tuboovarialabszess ist charakterisiert durch eine Ansammlung von Eiter im Inneren des Eileiters oder Ovars mit der Gefahr des Übergangs in eine Parametritis sowie eine Peritonitis. Diese Gefahr definiert den Bedarf einer notfallmäßigen Intervention in der Klinik. Pathogenetisch entsteht der Abszess als polymikrobielle Infektion auf dem Boden einer Pelvic Inflammatory Disease (PID; 1\% aller Frauen 15 - 39 Jahre). Die Symptomatik entwickelt sich über (Unter-)

- Der Verdacht auf Ovarialtorsion ergibt sich aus Anamnese, Klinik, Palpationsbefund und kann in der Klinik durch die sonografische Darstellung des aufgetriebenen Ovars ergänzt werden.

Ovarialtorsion ist für 3\% der gynäkologischen Notfalleingriffe, hiervon $15-25 \%$ in der Schwangerschaft, verantwortlich.
Der Tuboovarialabszess ist durch eine Ansammlung von Eiter im Inneren des Eileiters oder Ovars charakterisiert mit der Gefahr des Übergangs in eine Parametritis sowie eine Peritonitis. 


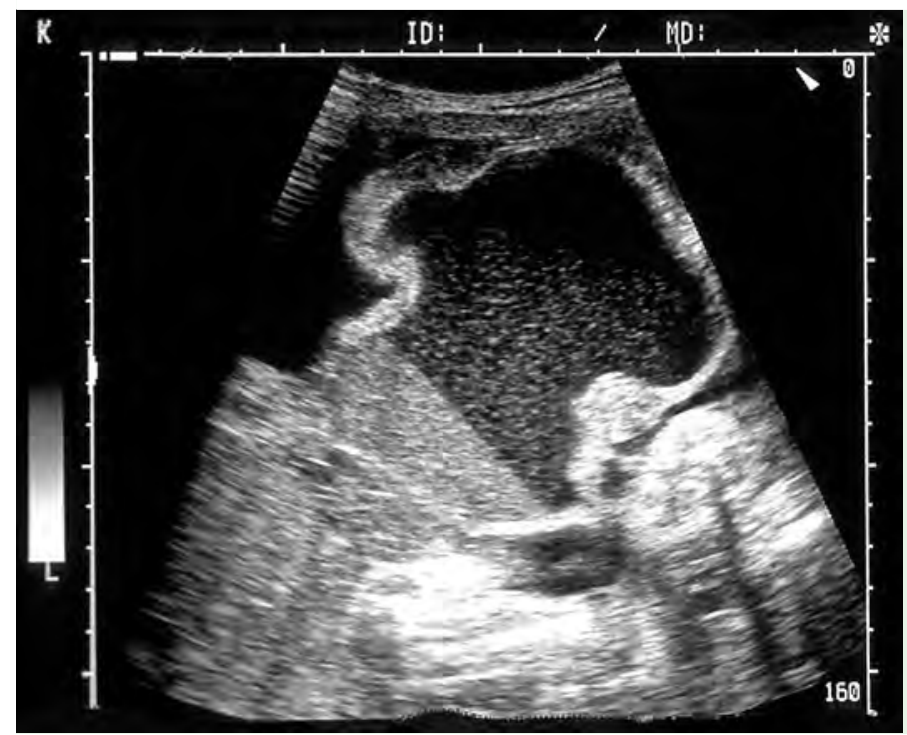

Abb.2 Tuboovarialabszess (Transvaginalsonografie): Scharf begrenzte, unregelmäßig konturierte zystische Raumforderung mit Spiegelbildung (diagnostisch hinweisend) im Adnexbereich.

Bauchbeschwerden als führendes Symptom (<90\%), Leukozytose $(60-80 \%)$, Fieber $(60-80 \%)$ bis hin zum akuten Abdomen. Die Diagnostik umfasst die klinische Untersuchung, Abstrichentnahme, biochemische Labordiagnostik (Entzündungsparameter), Körpertemperaturbestimmung und transvaginale Sonografie ( $\bullet$ Abb.2). Hinsichtlich der differenzialdiagnostischen Abgrenzung zu anderen Schmerzursachen ist an Appendizitis bzw. perityphilitischen Abszess, Extrauteringravidität, Endometriose, Ovarialtorsion, Ovarialvenenthrombose oder Malignom zu denken $[1,5,6]$. Die spezifische Behandlung des Tuboovarialabszesses erfolgt durch laparoskopische Abszessspaltung, Spülung und Drainage. Zeitgleich ist eine i.v. antibiotische Therapie der ursächlichen Keime (stets Chlamydien inkludieren - Doxycyclin) vorzunehmen. Bei promptem Ansprechen auf diese Maßnahmen ist die Prognose als gut zu werten. Eine palpable Raumforderung kann allerdings dennoch über mehrere Monate tastbar bleiben. Als Spätfolgen des Tuboovarialabszesses können Adhäsiones, Dysmenorrhö, Sterilität oder eine erhöhte Rate ektoper Schwangerschaften resultieren $[7,8]$.

\section{Ektope Schwangerschaft}

$\nabla$

Mögliche Lokalisationen einer ektopen Schwangerschaft sind der Eileiter, das Ovar, die freie Bauchhöhle und der Gebärmutterhalskanal.

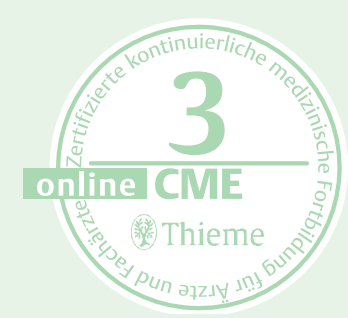

Jede Implantation einer befruchteten Eizelle außerhalb der Gebärmutterhöhle definiert eine ektope Schwangerschaft. Als Prädilektionsstellen kommen der Eileiter, das Ovar, die freie Bauchhöhle oder der Gebärmutterhalskanal infrage ( $\bullet$ Tab.2). Jede ektope Gravidität stellt durch das immanente Blutungsrisiko ein für die Frau potenziell lebensbedrohliches Ereignis dar [9-13].

Die Inzidenz des ektopen Sitzes beträgt 0,3 - 3\% aller Schwangerschaften. 99\% der Extrauteringraviditäten (EUG) sind im Eileiter lokalisiert ( $\mathbf{A b b}$.3). In den vergangenen Jahren ist eine deutliche Häufigkeitszunahme (Jahr 1970: 0,5\%; Jahr 1992: 1\%) durch eine Verfeinerung der diagnostischen Optionen, die zunehmende Häufung von Salpingitiden, die steigende Zahl von operativen Eingriffen an den Eileitern und einen Anstieg des mütterlichen Alters (<20 Jahre: 0,4\%; 20 - 29 Jahre: 0,7\%; 30 - 40 Jahre: 1,3-2\%) zu beobachten. Als wesentliche ätiologische Faktoren wirken sich im Einzelnen vorangehende Aborte oder ektope Graviditäten (30\%), (rezidivierende) Adnexitiden bzw. Appendizitis (25\%), Kinderwunschbehandlung - IVF (In-vitroFertilisation; 20\%), liegende Spirale (8- bis 10-fache Risikoerhöhung), Z.n. Tubensterilisation oder Tubenoperation und Endometriose aus. Das Wiederholungsrisiko beträgt $10 \%$ (nach wie-

Tab. 2 Häufigkeit ektoper Schwangerschaften in Abhängigkeit ihrer Lokalisation.

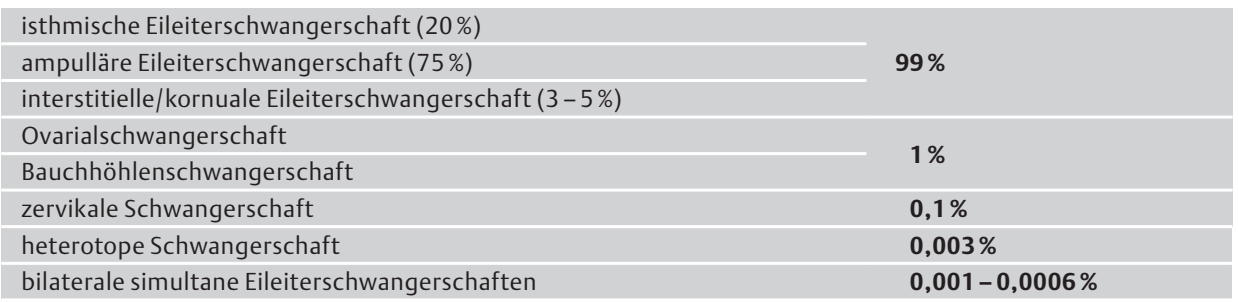




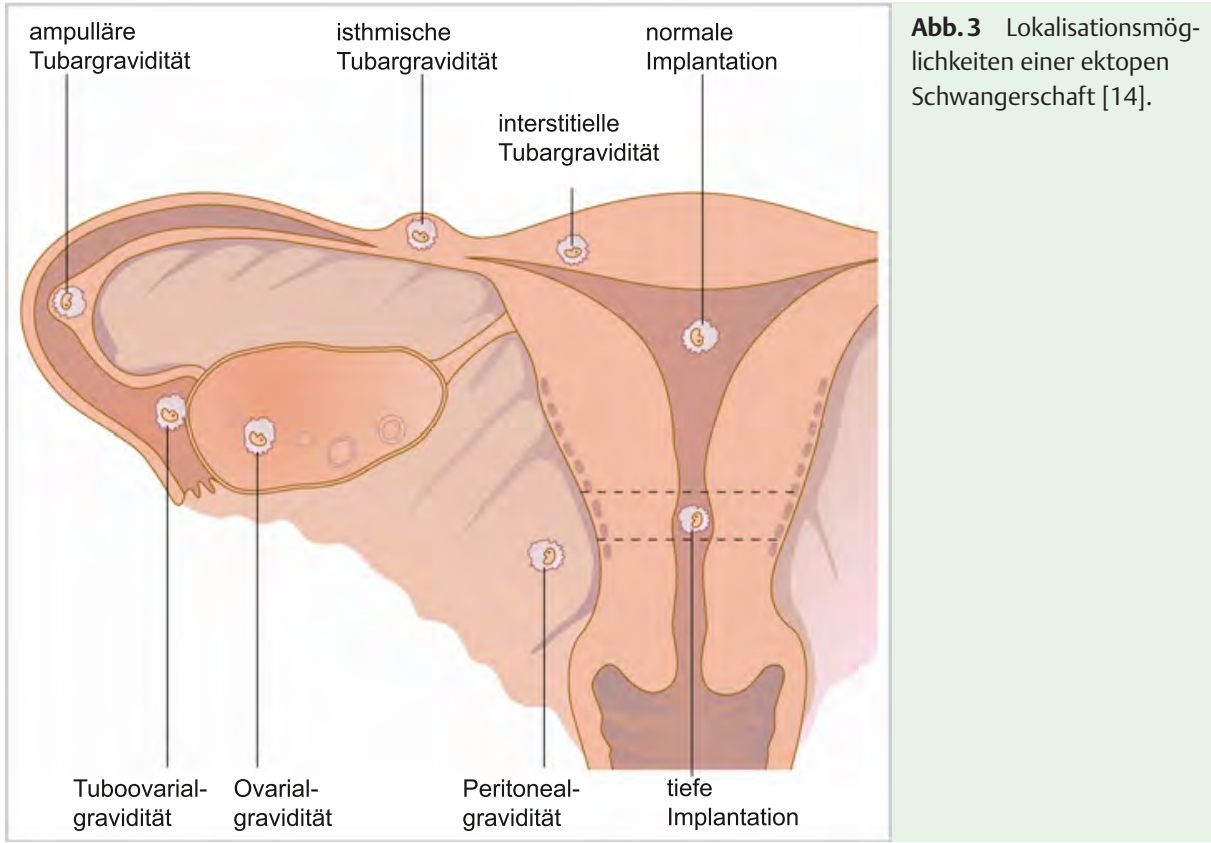

derholter EUG bis zu 50\%). Die Sterblichkeit ist im Gegensatz zur Inzidenz der Eileiterschwangerschaft durch Früherkennung, situationsadaptierte Therapie und verbesserte postoperative Überwachung deutlich rückläufig (1970er-Jahre: 1,7\%; Jahr 2005: 0,2\%). Trotzdem bleibt die ektope Schwangerschaft mit 0,0004\% (bezogen auf alle Schwangerschaften) die häufigste mütterliche Todesursache im 1. Schwangerschaftsdrittel (4,2 - 9\% aller mütterlichen Todesfälle).

\section{Tubarruptur}

Der klinische Verlauf einer Eileiterschwangerschaft entwickelt sich in Abhängigkeit ihrer Lokalisation stadienhaft: Das Stadium der intakten „stehenden“ Eileiterschwangerschaft (asymptomatische Patientin, Frucht und Eileiter intakt, positive Herzaktion des Embryos) wird im Fall einer Tubarruptur (=äußerer Fruchtkapselaufbruch) unmittelbar vom Stadium des peritonealen Schocks (perakute, heftigste abdominelle Zerreißungsschmerzen, Abwehrspannung, Kaltschweißigkeit, Schwächegefühl, Atemnot, Hypotonie, Tachykardie, bei größerer Blutungsmenge Phrenikusreizung - Oberbauch-, Schulter- oder Oberarmschmerz, akutes peritoneales hämorrhagisches Schockgeschehen) abgelöst ( $\bullet$ Abb.4).

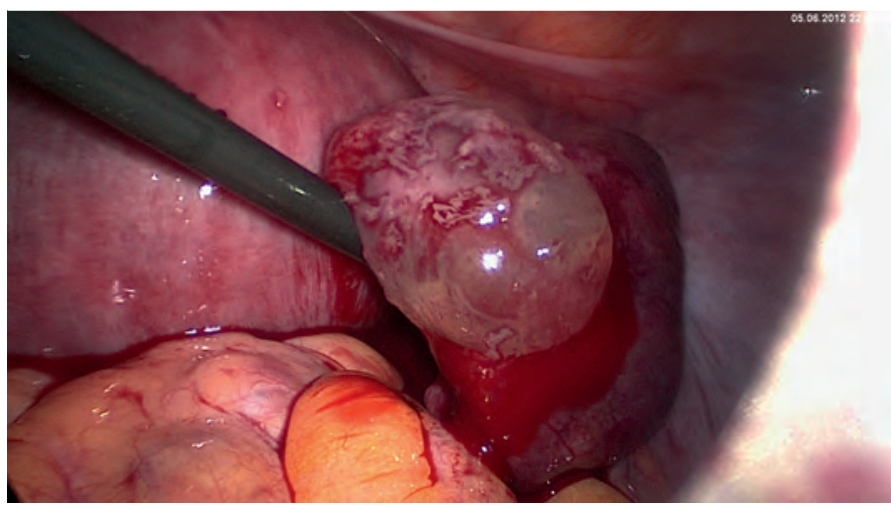

Abb. 4 Laparoskopischer Befund einer stehenden Eileiterschwangerschaft rechts (aufgetriebener ampullärer Eileiteranteil mit Fruchtsack mit „zystischer“ Fruchthöhle rechts unten und trophoblastären Plazentastrukturen links oben in der Abbildung).

\section{Tubarabort}

Ein Tubarabort (innerer Fruchtkapselaufbruch) entwickelt sich (6- bis 10-mal häufiger als eine Tubarruptur) durch Absterben und teilweise Ablösung des Trophoblasten von der Tubenwand (peritubares Hämatom, 1-seitig betonte wehenartige Schmerzattacken) langsamer und kann letztlich zum akuten Abdomen bei der Patientin führen. Seltene Sonderformen der ektopen Schwangerschaftslokalisation sind die interstitielle bzw. kornuale Schwangerschaft (intramuraler Eileiteranteil in der Gebärmutterwand), Ovarialgravidität, Bauchhöhlenschwangerschaft oder Zervixschwangerschaft $(\bullet$ Abb.5).

- Bei der Tubarruptur ist ein hämorrhagischer Schock bis hin zum Kreislaufstillstand innerhalb weniger Minuten möglich. 
Ausgeprägter Portioschiebeschmerz, eine im Douglas' schen Raum tastbare Hämatozele und die teigige, deutlich dolente Resistenz im Adnexbereich, bestimmen den klinischen Untersuchungsbefund eines Tumoraborts.
- Das Schwangerschaftshormon $\beta$-hCG spielt eine führende differenzialdiagnostische Rolle.

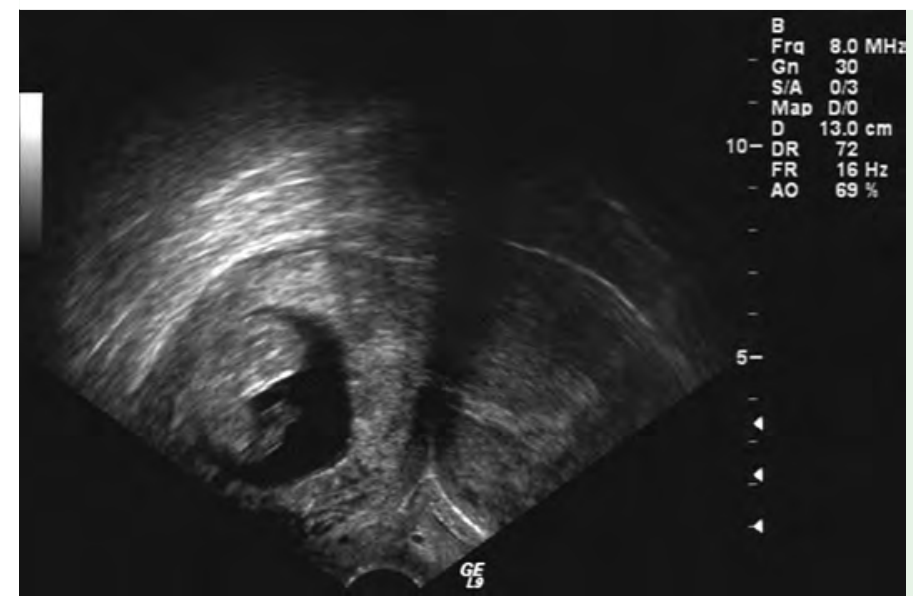

Abb. 5 Vaginalsonografie einer zervikal gelegenen Gravidität in der 13. SSW: abgegrenzte, echoleere Fruchthöhle mit Embryonalstruktur im Zervikalkanal des antevertierten, aufgelockerten Uterus (Gebärmutterkörper in der Abbildung rechts).
Neben den Akutsymptomen (Schmerzen, akutes Abdomen, Defense, hämorrhagischer bzw. peritonealer Schock) manifestiert sich die ektope Schwangerschaft durch sekundäre Amenorrhö (ca. 6-8 Wochen) bei positivem Schwangerschaftstest und irregulärer uteriner Schmierblutung. Ausgeprägter Portioschiebeschmerz, eine im Douglas'schen Raum tastbare Hämatozele und die teigige, deutlich dolente Resistenz im Adnexbereich, bestimmen den klinischen Untersuchungsbefund.

\section{Diagnose}

Die Diagnose wird anamnestisch, klinisch und laborchemisch gestellt.

\section{Anamnese}

- sekundäre Amenorrhö (6-8 Wochen); exakte Bestimmung der Amenorrhödauer ggf. durch Zwischenblutungen erschwert

- gynäkologische Vorerkrankungen (v.a. Adnexitiden)

- Operationen (v.a. Sterilisation)

- Schwangerschaften (u.a. frühere EUG)

- Geburten

\section{Klinische Untersuchung}

- ggf. vaginale (Schmier-)Blutung

- ausgeprägter Portioschiebeschmerz

- Tubenbereich - heftig schmerzhafter teigiger Tumor

- abdominale Palpation - Abwehrspannung

\section{Serologie}

Die führende differenzialdiagnostische Rolle spielt die Bestimmung des Schwangerschaftshormons $\boldsymbol{\beta}$-hCG (hCG: humanes Choriongonadotropin; positiv ab 10-14 Tage post conceptionem). Zur schnellen semiquantitativen Diagnose einer Schwangerschaft wird hierzu ein Schwangerschaftsschnelltest im Urin (ggf. quantitativ auch im Serum) verwendet. Der früheste Nachweis gelingt im mütterlichen Serum bereits 10-14 Tage nach der Konzeption (Nachweisgrenze: 5 I.E./l). Bei intrauterinen Schwangerschaften verdoppelt sich die $\beta$-hCG-Konzentration im Serum zunächst etwa alle 2 Tage (bis 40 Tage post menstruationem). In ektopen Schwangerschaften erfolgt der Anstieg langsamer. Abfallende oder nicht adäquat ansteigende $\beta$-hCG-Werte begründen bzw. bestärken daher die Verdachtsdiagnose.

\section{Sonografie}

Daneben ist die sonografische Diagnostik die bildgebende Methode der Wahl (ab $\beta$-hCG 1000 1500I.E./l gelingt die Darstellung der Chorionhöhle mit einer Sensitivität von $90 \%$ und einer Spezifität von 98\%). Meist sind es allerdings indirekte sonografische Hinweiszeichen, die zur Diagnose (fehlende Darstellung einer intrauterinen Schwangerschaft, Pseudogestationssack, [unklare] Raumforderung im Adnexbereich, Darstellung von Embryo und bzw. oder Dottersack [5-10\%], freie intraabdominelle Flüssigkeit [30-90\%]) führen. 


\section{Differenzialdiagnosen}

- (schmerzhafte) intrauterine Schwangerschaft

- intrauteriner Abort

- zystischer Adnextumor (Corpus luteum) ggf. mit peritonealer Reizung (z. B. Ruptur)

$\checkmark$ entzündliche Adnexerkrankung, Hydro-, Pyo-, Hämatosalpinx, Tuboovarialabszess

- Appendizitis (typischer rechtsseitiger Schmerz - McBurney-Punkt)

- ovarielles Überstimulationssyndrom (Ovarialtumore und Aszites)

\section{Therapie}

Für die präklinische Versorgung ergibt sich abhängig vom Blutdruck der Patientin Interventionsbedarf. Analog den Empfehlungen für Traumapatienten mit Volumenmangel sollte ein Zielblutdruck von $90 \mathrm{~mm} \mathrm{Hg}$ aufrechterhalten oder erreicht werden.

Bei vitaler Bedrohung - Schock - ist in der Klinik eine sofortige Bestimmung des Hämoglobinwerts, der Gerinnungsparameter, wenn erforderlich die Verabreichung von Blutprodukten (ggf. zunächst ungekreuzte Notfallkonserven) und die umgehende Operation der Patientin zwingend. Bereits bei Verdacht auf die Akutsymptomatik einer ektopen Schwangerschaft ist daher der umgehende Transport in eine entsprechend geeignete Klinik erforderlich.

Die Wahl der Behandlungsmethode wird vom klinischen Bild, der hämodynamischen Situation, dem $\beta$-hCG-Wert und dem Wunsch der Patientin nach weiteren Schwangerschaften bestimmt: Expektatives Vorgehen (Symptomfreiheit, negativer oder diskreter Ultraschallbefund, niedrige und fallende $\beta$-hCG-Werte < 300 I.E./I), chirurgische Therapie (laparoskopische bzw. laparotomische Salpingotomie, Keilexzision aus dem Eileiter oder Salpingektomie) oder medikamentöse Therapie (Methotrexat systemisch i.m. oder i.v. $50 \mathrm{mg} / \mathrm{m}^{2}$ Körperoberfläche bzw. $1 \mathrm{mg} / \mathrm{kg}$ Körpergewicht oder lokal $10-50 \mathrm{mg}$ ) sind dabei die Alternativen. Der Einsatz von konservativen Behandlungsansätzen bedarf nach entsprechender Aufklärung einer patientenzentrierten Abwägungsentscheidung. Die Überwachung des Therapieansprechens (ambulant oder stationär) erfolgt durch das Monitoring des $\beta$-hCG-Abfalls ( $<15 \%$ zwischen Tag 4 und 7 ). Hinsichtlich des operativen Vorgehens hat der minimalinvasive laparoskopische Ansatz in den vergangenen Jahrzehnten die Laparotomie sowohl in der Diagnostik als auch in der operativen Behandlung der EUG weitgehend verdrängt.

Bei jeder Form der Behandlung einer ektopen Gravidität (expektativ, operativ oder medikamentös) ist die engmaschige sonografische und laborchemische Begleitung (bis $\beta$-hCG < 5 I.E./l), u.U. für 4-6 Wochen, zur Sicherung des Therapieerfolgs obligat.

Unabhängig der Therapiemodalität ist jeder nicht sensibilisierten Rhesus-negativen Patientin, analog zur intrauterinen Fehlgeburt, posttherapeutisch eine Anti-D-Prophylaxe zu verabreichen [15].

\section{Präklinische Spezifika im Umgang mit einer gynäkologischen Notfallpatientin}

$\checkmark$

Insbesondere in der klinischen Gynäkologie kommt der Ultraschalluntersuchung (v.a. transvaginal) zur Diagnostik bei gynäkologischen Notfallpatientinnen eine überragende Bedeutung zu. Da dieses Diagnostikum in der präklinischen Notfallsituation nicht zur Verfügung steht, sollte hier differenzialdiagnostisch besonderes Augenmerk auf die Anamnese gerichtet werden. In Abhängigkeit dieser Einschätzung erfolgt die Auswahl einer geeigneten Zielklinik.

Zentrale Fragen, abgestimmt auf die gynäkologische Situation sind:

- Wann war die letzte Periode? Ist der Menstruationszyklus regelmäßig?

mittzyklisch und Unterbauchschmerz $\rightarrow$ ggf. Ovarialzystenruptur

Periode unregelmäßig $\rightarrow$ ggf. Schwangerschaft?

- Hat die Frau Kenntnis von einer Schwangerschaft? Auch wenn die Patientin eine Schwangerschaft verneint, sollte bei prämenopausalen Patientinnen im Verlauf immer ein Schwangerschaftstest erfolgen $\rightarrow$ intakte intrauterine Schwangerschaft, DD (Differenzialdiagnose) gestörte intrauterine Schwangerschaft, DD ektope Schwangerschaft

- Bestehen unsichere Schwangerschaftszeichen (Brustspannen, morgendliche Übelkeit)? $\rightarrow$ intakte intrauterine Schwangerschaft, DD gestörte intrauterine Schwangerschaft, DD ektope Schwangerschaft

- Wie ist der Schmerz der Patientin charakterisiert? krampfartig und kurz vor Periode bzw. mit Einsetzen der Periode $\rightarrow$ Dysmenorrhö akut und Amenorrhö $\rightarrow$ ggf. intrauterine (intakte/gestörte) oder ektope Schwangerschaft akut messerstichartig beginnend, ggf. mittzyklisch $\rightarrow$ Ovarialzystenruptur bzw. -einblutung plötzlich beginnend, ggf. schmerzfreies Intervall, vegetative Begleitsymptomatik $\rightarrow$ Ovarial (-zysten)torsion

Bei vitaler Bedrohung - Schock - ist in der Klinik eine sofortige Bestimmung des Hämoglobinwerts, der Gerinnungsparameter, wenn erforderlich die Verabreichung von Blutprodukten (ggf. zunächst ungekreuzte Notfallkonserven) und die umgehende Operation der Patientin zwingend.

- Bei jeder Form der Behandlung einer ektopen Gravidität (expektativ, operativ oder medikamentös) ist die engmaschige sonografische und laborchemische Begleitung, u.U. für 4-6 Wochen, zur Sicherung des Therapieerfolgs obligat. 
Neben der Anamnese erfolgt wie bei jeder Notfallpatientin eine strukturierte Untersuchung und eine prioritätenorientierte Versorgung (ABCDE-Schema). Befindet sich die Patientin in einem Volumenmangelschock, so sollten neben der Sauerstoffinsufflation auch mindestens 2 großlumige Gefäßzugänge etabliert werden. Die Infusionstherapie und Auswahl der Infusionslösungen orientiert sich an den Leitlinien zur Polytraumaversorgung. Gerade bei durch den Notarzt nicht stillbaren Blutungssituationen werden analog die Empfehlungen zur Traumaversorgung bei stumpfen Traumata genutzt. Hierbei werden hypotone Blutdruckwerte bis $90 \mathrm{~mm} \mathrm{Hg}$ akzeptiert. Die Analgesie erfolgt mit den sonst auch im Notarztdienst üblichen Medikamenten (s. [16]). Gerade bei starken und stärksten Schmerzzuständen kommen Opiate (z.B. Piritramid oder Morphin) zur Anwendung. Als Zielkrankenhaus sollte bei vaginaler Blutung oder Patientinnen im gebährfähigen Alter mit Unterbauchschmerzen eine Klinik mit gynäkologischer Fachabteilung primär angefahren werden. Bei akuter intraabdominaler Blutung und instabilen Kreislaufparametern sollte die nächsterreichbare Klinik mit chirurgischer Interventionsmöglichkeit das Transportziel sein. Wie bei allen Notfallpatienten mit einem Bedarf für eine sofortige operative Intervention sollte die entsprechend ausgewählte Zielklinik so rechtzeitig wie möglich über die bevorstehende Zuweisung informiert werden. Zur sicheren Übermittlung der notwendigen medizinischen Informationen hat sich neben der Anmeldung über die Rettungsleitstelle ein direktes Arzt-Arzt-Gespräch bewährt.

\section{Interessenkonflikt \\ $\nabla$}

Im Zusammenhang mit dem vorliegenden Artikel bestehen keine Interessenkonflikte für Alexander Strauss, Lea Sanders, Jan-Thorsten Gräsner und Henning Ohnesorge.

\section{Literatur}

1 Strauss A, Janni W, Maass N. Klinikmanual Gynäkologie und Geburtshilfe. 1. Aufl. Heidelberg: Springer; 2009

2 Strauss A, Kümper C, Bauer M et al. Vaginale Blutungen in der Gynäkologie. Gyne 2010; 31: 20

3 AWMF Standards zur Ultraschalluntersuchung in der Frühschwangerschaft. 2001. Im Internet: http:// www.awmf.org/ (Stand: 2013)

4 Strauss A. Ultraschallpraxis Geburtshilfe und Gynäkologie. 2. Aufl. Heidelberg: Springer; 2008

5 Strauss A, Meinhold-Heerlein I, Kümper C. Notfälle in Gynäkologie und Geburtshilfe. Notfallmed up2date 2008; 3: 237-255

6 Strauss A, Meinhold-Heerlein I, Heer IM et al. Notfälle in der Frauenheilkunde. Geburtsh Frauenheilkd 2010; 70: R21-44

7 Strauss A, Zabel F. Gynäkologische Notfälle. In: Domschke W, Berger M, Hohenberger W, Meinertz T, Possinger K, Hrsg. Therapiehandbuch. 5. Aufl. München: Elsevier, Urban \& Fischer; 2011

8 Strauss A, Heer IM, Kümper C. Gynäkologische und geburtshilfliche Notfälle. In: Fleischmann T, Hrsg. Klinische Notfallmedizin. 1. Aufl. München, Jena: Elsevier, Urban \& Fischer; 2011

9 Kuçera E, Lehner R, Husslein P. Extrauteringravidität. In: Schneider HPG, Husslein P, Schneider KTM, Hrsg. Die Geburtshilfe. 1. Aufl. Heidelberg: Springer; 2003

10 Strauss A, Bauer M, Weigel MT et al. Die ektope Gravidität. Gyne 2010; 5: 10 - 16

11 Kainer F. Ektope Schwangerschaft. In: Dudenhausen JW, Schneider HPG, Hrsg. Frauenheilkunde und Geburtshilfe. 18. Aufl. Berlin: Walter de Gruyter; 1994

12 American Society of Reproductive Medicine. Practice Guidelines in early diagnosis and management of Ectopic Pregnancy. Im Internet: http://www.asrm.org/uploadedFiles/ASRM_Content/Resources/Patient_ Resources/Fact_Sheets_and_Info_Booklets/ectopicpregnancy_B.pdf (Stand: 2006)

13 Dudenhausen JW, Pschyrembel W. Extrauteringravidität, Tubargravidität. In: Praktische Geburtshilfe: mit geburtshilflichen Operationen. 19. Aufl. Berlin: Walter de Gruyter; 2001

14 Lermann J, Müller A, Burghaus S et al. Praxistipp Ultraschall und operative Therapie. Die Extrauteringravidität. Geburtsh Frauenheilk 2012; 72: 116-121

15 RCOG Clinical Green Top Guidelines: The management of tubal pregnancies (21). 1999

16 Strauss A, Sanders L, Ohnesorge H et al. Geburtshilfliche Notfälle III - Präklinische Geburtshilfe. Der Notarzt 2013; 29: 115-126

17 Strauss A. Ektope Schwangerschaft. In: Kainer F, Hrsg. Facharzt Geburtsmedizin. 2. Aufl. München, Jena: Elsevier, Urban \& Fischer; 2012

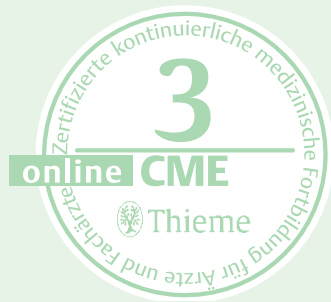

18 Korell M. Extrauteringravidität. In: Nestle-Krämling C, Korell M, Hrsg. Bildatlas der Patientinnenaufklärung Gynäkologie. 1. Aufl. Balingen: Spitta; 2003 


\section{CME-Fragen Gynäkologische Notfälle}

A Bei prämenopausalen Patientinnen sollte an ein Abortgeschehen gedacht werden.

B Bei starker vaginaler Blutung kommt u.U. bereits präklinisch dem Volumenersatz hohe Bedeutung zu.

C Bei kreislaufstabiler Patientin können, in der Regel unter stationären Bedingungen, medikamentöse Therapieoptionen eingesetzt werden.

D Malignome kommen als Ursache vaginaler Blutungen nicht in Betracht.

E Im Verhältnis zur Sicherung der Vitalparameter und der ggf. erforderlichen Unterstützung der Kreislaufparameter der Patientin kommt der kausalen Behandlung der Blutungsursache in der präklinischen Akutsituation eine untergeordnete bzw. keine Bedeutung zu.

Welche Aussage zu Ovarialzysten ist nicht richtig?

A Einblutung oder Zystenruptur können zu einem akuten Schmerzereignis führen.

B Dem Schmerzereignis liegt üblicherweise eine peritoneale Reizung zugrunde.

C Bei V.a. rupturierte Ovarialzyste muss nach Klinikeinweisung immer operiert werden.

D Typisch ist ein akut einsetzender Schmerz in der Zyklusmitte.

E Eine Ovarialzystenruptur kann auch gänzlich asymptomatisch verlaufen.

Welche Aussage zur ovariellen Torsion trifft zu?

A Die Ovartorsion ist eine häufige gynäkologische Ursache von Unterbauchschmerzen.

B Bei einer Ovartorsion kann es zum Absterben des betreffenden Ovars kommen.

C Schon präklinisch kann die Diagnose einer Ovartorsion anhand ihrer Klinik stets sichergestellt werden.

D Bei V.a. Ovartorsion ist ein abwartendes Vorgehen indiziert.

E Die Ovartorsion geht üblicherweise nicht mit vegetativen Begleitsymptomen wie Übelkeit und Erbrechen einher.

\section{Welche Aussage zum Tuboovarialabszess trifft nicht zu?}

A Tuboovarialabszesse treten nur in der Postmenopause auf.

B Die Ovartorsion ist eine der gynäkologischen Differenzialdiagnosen des Tuboovarialabszesses.

C Eine antibiotische Therapie (in der Klinik) ist bei dieser Erkrankung geboten.

D Ein Tuboovarialabszess stellt eine Indikation zur operativen Therapie dar.

E Fieber tritt bei dieser entzündlichen Erkrankung häufig auf.

\section{Welche Aussage zur Extrauteringravidität ist richtig?}

A Die Inzidenz der Extrauteringravidität ist in den vergangenen Jahren deutlich rückläufig.

B Jede Implantation einer befruchteten Eizelle außerhalb der Gebärmutterhöhle definiert eine ektope Schwangerschaft.

C Die häufigste Lokalisation einer ektopen Schwangerschaft ist die Cervix uteri.

D Die häufigste Lokalisation einer ektopen Schwangerschaft ist die freie Bauchhöhle.

E Eine Extrauteringravidität ist stets klinisch zu diagnostizieren.

6

Welche Aussage zur Extrauteringravidität ist nicht richtig?

A Jede Extrauteringravidität stellt durch das Blutungsrisiko ein für die Frau potenziell lebensbedrohliches Ereignis dar.

B Die Symptome der Extrauteringravidität können perakut unter dem Bild eines akuten Abdomens auftreten.

C Risikofaktor einer Extrauteringravidität ist der Zustand nach Salpingitis bzw. Tuboovarialabszess.

D Risikofaktor einer Extrauteringravidität ist eine durchgeführte Kinderwunschbehandlung (IVF).

E Die rupturierte Eileiterschwangerschaft stellt keine unmittelbare Notfallsituation dar. Eine operative Therapie ist nicht dringlich durchzuführen.

7

A

aufweisen.

B präklinisch ohne Analgetika versorgt werden, um die Diagnostik in der Klinik zu erleichtern.

C analog den Empfehlungen für stumpfe Traumata gemäß der S3-Leitlinie zur Polytraumaversorgung versorgt werden.

D zur Diagnostik immer ein CT-Abdomen erhalten.

E ausschließlich in gynäkologischen Fachkliniken stationär versorgt werden.

8 Welche Diagnostik ist präklinisch bei Verdacht auf intraabdominelle Blutungen sinnvoll? vaginale Untersuchung transvaginaler Ultraschall klinische Untersuchung gemäß ABCDE-Schema rektale Untersuchung Loslassschmerz-Diagnostik am McBurney-Punkt

9 Welche präklinische Maßnahme ist grundsätzlich bei Patientinnen mit Verdacht auf eine gynäkologische Notfallursache entbehrlich?

A vaginale gynäkologische Untersuchung

B Anamneseerhebung

C klinische Untersuchung des Abdomens

D Kontrolle des Blutdrucks

E Kontrolle des Pulses 
B keine Analgetika, da sie die Diagnostik in der Klinik erschweren

C Paracetamol rektal

D Opiate intravenös

E Diclofenac oral

\section{Impressum CME - Der Notarzt}

\section{(c) 2013 Georg Thieme Verlag KG}

Rüdigerstr. 14, 70469 Stuttgart

Unsere Homepage:

http://www.thieme.de

Printed in Germany

Satz: Maisch, Ditzingen

Druck: F\&W Mediencenter GmbH, Kienberg

Redaktionelle Mitarbeit:

Sabine M. Rüdesheim,

Frechen-Königsdorf

\section{Ansprechpartner:}

Dr. Marion Rukavina

Redaktion

Georg Thieme Verlag KG

Klinik und Praxis

E-Mail: Marion.Rukavina@thieme.de

\section{Wichtige Hinweise}

Die CME-Beiträge der Refresher Der Notarzt wurden durch die Nordrheinische Akademie für ärztliche Fort- und Weiterbildung anerkannt. Der Notarzt ist zur Vergabe der Fortbildungspunkte für diese Fortbildungseinheit berechtigt. Diese Fortbildungspunkte der Nordrheinischen Akademie für ärztliche Fort- und Weiterbildung werden von den anderen zertifizierenden Ärztekammern sowie, gemäß der Novellierung der DFP-Richtlinien vom 30.6.2010 (§14 Ziff. 3), auch von den österreichischen Ärztekammern anerkannt. Die Vergabe der Fortbildungspunkte ist nicht an ein Abonnement gekoppelt!

Für Angaben über Dosierungsanweisungen und Applikationsformen kann vom Verlag keine Gewähr übernommen werden. Jeder Benutzer ist angehalten, durch sorgfältige Prüfung der Beipackzettel der verwendeten Präparate und ggf. nach Konsultation eines Spezialisten festzustellen, ob die dort gegebene Empfehlung für Dosierungen oder die Beachtung von Kontraindikationen gegenüber der Angabe in diesem Beitrag abweicht. Eine solche Prüfung ist besonders wichtig bei selten verwendeten Präparaten oder solchen, die neu auf den Markt gebracht worden sind. Jede Dosierung oder Applikation erfolgt auf eigene Gefahr des Benutzers.

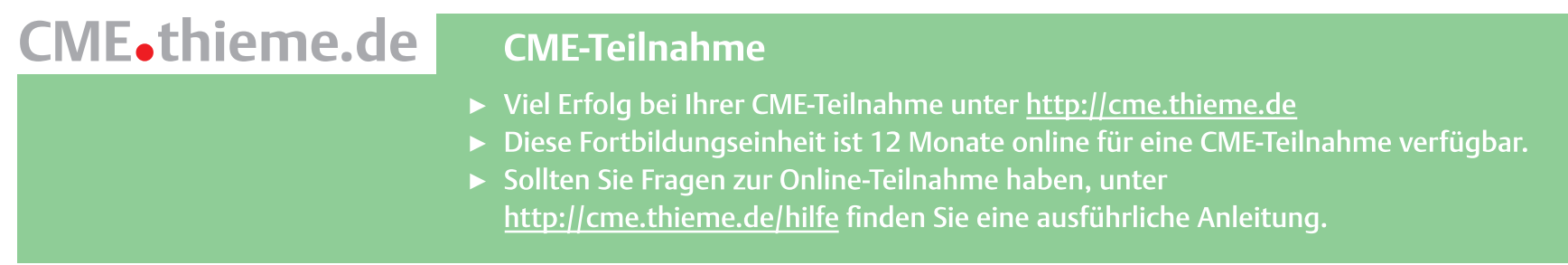

